Pacific Journal of Mathematics

ON THE STRUCTURE OF INFRAPOLYNOMIALS WITH OVEn SWISH 


\section{ON THE STRUCTURE OF INFRAPOLYNOMIALS WITH PRESCRIBED COEFFICIENTS}

\section{O. SHISHA}

Introduction. The main result of this paper is Theorem 5 which deals with the structure of infrapolynomials with prescribed coefficients. This theorem was quoted (without proof) in a previous paper [Shisha and Walsh, 1961] $]^{1}$, and was used there to prove a few results concerning the geometrical location of the zeros of some infrapolynomials with prescribed coefficients [loc. cit., Theorems 11, 12, 16, 17]. Two similar results are given here in Theorem 6 .

We refer the reader to the Introduction of the last mentioned paper for a review of the development of the concept of infrapolynomial. Here we shall just mention two of the underlying definitions.

A. Let $n$ and $q$ be natural numbers $(q \leq n), n_{1}, n_{2}, \cdots, n_{q}$ integers such that $0 \leq n_{1}<n_{2} \cdots<n_{q} \leq n$, and $S$ a set in the complex plane ${ }^{2}$. An $n$th infrapolynomial on $S$ with respect to $\left(n_{1}, n_{2}, \cdots, n_{q}\right)$ is a polynomial $A(z) \equiv \sum_{\nu=0}^{n} a_{\nu} z^{\nu}$ such that no $B(z) \equiv \sum_{\nu=0}^{n} b_{\nu} z^{\nu}$ exists, satisfying the following properties.

(1) $B(z) \not \equiv A(z)$,

(2) $b_{n_{\nu}}=a_{n_{\nu}}(\nu=1,2, \cdots, q)$,

(3) $|B(z)|<|A(z)|$ whenever $z \in S$ and $A(z) \neq 0$, and

(4) $B(z)=0$ whenever $z \in S$ and $A(z)=0$.

B. Let $n$ be a natural number. $A$ simple $n$-sequence is a sequence having one of the forms

$$
\begin{gathered}
(0,1, \cdots, k, n-l, n-l+1, \cdots, n)[k \geq 0, l \geq 0, k+l+2 \leq n], \\
(0,1, \cdots, k)[0 \leq k<n],(n-l, n-l+1, \cdots, n)[0 \leq l<n] .
\end{gathered}
$$

Theorem 5 may yield information on the location of the zeros of an $n$th infrapolynomial $A(z)$ on a set $S$ with respect to a simple $n$ sequence $\sigma$. For it allows (under quite general conditions) to set $A(z) \equiv B(z) D(z)$ where $D(z)$ is a polynomial all of whose zeros lie in $S$, whereas $B(z)$ is a divisor of a polynomial $Q(z)$ whose structure is given by the theorem. By studying the location of the zeros of $Q(z)$, one may get information on the location of the zeros of $A(z)$. By this method, Theorems 11, 12, 16, 17 [loc. cit.] were proved. (Compare

Received September 12, 1963. This research was supported (in part) by the U.S Air Force through the Air Force Office of Scientific Research.

1 Dates in square brackets refer to the bibliography.

2 We deal throughout this paper with the open plane of complex numbers. 
also the proof of Theorem 6 below.)

Theorem 5 is a generalization of Fekete's structure theorem [1951], and we use his method of proof [cf. also Fekete 1955]. The concept of a "juxtafunction" (Definition 1) is a generalization of Fekete's "nearest polynomial" [1955], later termed "juxtapolynomial" [Walsh and Motzkin 1957]. Theorems 1-4 and Lemmas 1-4 are contained in the author's $\mathrm{Ph}$. D. thesis [1958]; they are needed for the proof of Theorem 5, and they generalize previous results of Fekete [1951, 1955]. The principal results of the present paper were published by the author (without proof) in abstracts (1958a, 1959, 1961].

1. Definition 1. Let $S$ be a set in the complex plane and let $\Pi$ be a set of complex functions defined on ${ }^{3} S$ such that whenever $f_{1} \in \Pi$, $f_{2} \in \Pi$ and $c_{1}, c_{2}$ are complex numbers, then $c_{1} f_{1}+c_{2} f_{2} \in I$. Let $f$ be a complex function defined on $S$. A juxtafunction to $f$ on $S$ with respect to $I I$ is an element $p$ of $\Pi$ having the property: there does not exist a $q \in \Pi$ satisfying

(a) $q(z) \neq f(z)$ for at least one $z \in S$,

(b) $|f(z)-q(z)|<|f(z)-p(z)|$ whenver $z \in S$ and $p(z) \neq f(z)$,

(c) $q(z)=f(z)$ whenever $z \in S$ and $p(z)=f(z)$.

Examples A. Let $S(\neq \varnothing)$ be $^{5}$ a closed and bounded set in the complex plane. Let $f, p_{1}, p_{2}, \cdots, p_{n}, \mu$ be complex functions with domain $S$ which are continuous on $S$, and assume, furthermore, that $\mu(z) \neq 0$ throughout $S$. For every complex function $\psi$ with domain $S$ which is continuous on $S$, let $\|\psi\|=\max [|\mu(z) \psi(z)|, z$ on $S]$. It is known that there exist complex numbers $\lambda_{1}^{*}, \lambda_{2}^{*}, \cdots, \lambda_{n}^{*}$ such that for every complex $\lambda_{1} \lambda_{2}, \cdots, \lambda_{n}$,

$$
\left\|f-\sum_{\nu=1}^{n} \lambda_{\nu}^{*} f_{\nu}\right\| \leq\left\|f-\sum_{\nu=1}^{n} \lambda_{\nu} f_{\nu}\right\| .
$$

Consider the linear space $I I$ of all linear combinations (with complex coefficients) of $p_{1}, p_{2}, \cdots, p_{n}$. Then $p=\sum_{\nu=1}^{n} \lambda_{\nu}^{*} p_{\nu}$ is a juxtafunction to $f$ on $S$ with respect to $\Pi$. Indeed, suppose that some $q=\sum_{\nu=1}^{n} \lambda_{\nu}^{\prime} p_{\nu}$ satisfies (a), (b) and (c) of Definition 1. Let $\zeta$ be a point of $S$ such that

$$
\|f-q\|=|\mu(\zeta)(f(\zeta)-q(\zeta))| .
$$

Then by (a) $q(\zeta) \neq f(\zeta)$, and therefore, by (c), $p(\zeta) \neq f(\zeta)$. From (b) we get $\left\|f-\sum_{\nu=1}^{n} \lambda_{\nu}^{\prime} f_{\nu}\right\|=\|f-q\|=|\mu(\zeta)(f(\zeta)-q(\zeta))|<\mid \mu(\zeta)(f(\zeta)$

\footnotetext{
3 i.e. their domains include $S$.

4 The domain of $c_{1} f_{1}+c_{2} f_{2}$ is the intersection of those of $f_{1}$ and $f_{2}$.

$5 \varnothing$ denotes the empty set.
} 


\section{$-p(\zeta)) \mid \leq\|f-q\|=\left\|f-\sum_{\nu=1}^{n} \lambda_{\nu}^{*} f_{\nu}\right\|$, contradicting (1).}

B. Let $f, p_{1}, p_{2}, \cdots, p_{n}$ be real functions with domain $S=[0,1]$, continuous there, and assume furthermore that $p_{1}, p_{2}, \cdots, p_{n}$ are orthonormal on $[0,1]$. Let $I$ be again the set of all linear combinations (with complex coefficients) of $p_{1}, p_{2}, \cdots p_{n}$. Let $\lambda_{\nu}^{*}=\int_{0}^{1} f(x) p_{\nu}(x) d x$ $(\nu=1,2, \cdots, n)$. Then $p=\sum_{\nu=1}^{n} \lambda_{\nu}^{*} p_{\nu}$ is a juxtafunction to $f$ on $S$ with respect to $\Pi$. Indeed, if $p=f$, then the last assertion follows from Lemma 1 below. We thus assume that $p\left(x_{0}\right) \neq f\left(x_{0}\right)$ for some $x_{0} \in[0,1]$. Suppose there exists a $q=\sum_{\nu=1}^{n} \lambda_{\nu} p_{\nu}$ satisfying (a), (b) and (c) of Definition 1. Then $|f(x)-q(x)| \leq|f(x)-p(x)|$ throughout $[0,1]$, and $\left|f\left(x_{0}\right)-q\left(x_{0}\right)\right|<\left|f\left(x_{0}\right)-p\left(x_{0}\right)\right|$. Thus

$$
\int_{0}^{1}\left[f(x)-\sum_{\nu=1}^{n} \operatorname{Re}\left(\lambda_{\nu}\right) p_{\nu}(x)\right]^{2} d x<\int_{0}^{1}\left[f(x)-\sum_{\nu=1}^{n} \lambda_{\nu}^{*} p_{\nu}(x)\right]^{2} d x,
$$

contradicting the least squares property of the Fourier coefficients $\lambda_{\nu}^{*}$.

Lemma 1. Let $S$ and $\Pi$ be as in Definition 1 and let $f$ be an element of $I$ with domain $S$. Then $f$ is the unique function with domain $S$ which is a juxtafunction to $f$ on $S$ with respect to $I$.

Proof. $f$ is such a juxtafunction, since (a) and (c) of Definition 1 are mutually contradictory when $p$ is $f$. If $p$ (with domain $S$ ) belongs to $\Pi$ and $p \neq f$, then $q=\frac{1}{2}(p+f)$ belongs to $\Pi$ and satisfies (a), (b) and (c), so that $p$ is not a juxtafunction to $f$ on $S$ with respect to $\Pi$.

\section{THEOREM 1.}

\section{Hypotheses.}

1. $S(\neq \varnothing)$ is a closed and bounded set in the complex plane, $f, p_{1}$, $p_{2}, \cdots, p_{n}$ are complex functions defined and continuous on $^{6} S$.

2. II is the set of all complex functions defined on $S$ which can be represented throughout $S$ as linear combinations (with complex coefficients) of the $p_{\nu}^{\prime} s$.

3. $p$ is a juxtafunction to $f$ on $S$ with respect to $\Pi$, and $p(z)$ $\neq f(z)$ throughout $S$.

6 As the domain of $f$ may properly include $S$, its continuity on $S$ means that if $a \in S$, and if $\left(a_{j}\right)_{j=1}^{\infty}$ is a sequence of points of $S$ converging to $a$, them $\lim _{j \rightarrow \infty} f\left(a_{j}\right)=f(a)$. Similarly for $p_{1}, p_{2}, \cdots, p_{n}$ and in Lemma 2 . 
Conclusion. There exist distinct points $z_{1}, z_{2}, \cdots, z_{m}$ of $S(1 \leq m$ $\leq 2 n+1)$ and positive $\lambda_{1}, \lambda_{2}, \cdots, \lambda_{m}$ such that:

(I). $p(z)$ is a juxtafunction to $f$ on $s=\left\{z_{1}, z_{2}, \cdots, z_{m}\right\}$ with respect to $\Pi$,

(II). No complex $b_{1}, b_{2}, \cdots, b_{n}$ exist such that $\left|f(z)-\sum_{\nu=1}^{n} b_{\nu} p_{\nu}(z)\right|<$ $|f(z)-p(z)|$ throughout $s$,

(III). $\quad \sum_{\mu=1}^{m} \lambda_{\mu} p_{\nu}\left(z_{\mu}\right) /\left\{f\left(z_{\mu}\right)-p\left(z_{\mu}\right)\right\}=0, \nu=1,2, \cdots, n$.

REMARK 1. Observe that (I) is implied by (II).

For the proof of Theorem 1 we shall need two lemmas.

LEMMA 2. Let $S(\neq \varnothing)$ be a closed and bounded set in the complex plane, and $\Pi$ a set of complex functions, defined and continuous on $S$ such that whenever $f_{1} \in \Pi, f_{2} \in \Pi$, and $c_{1}$ and $c_{2}$ are complex numbers, then $c_{1} f_{1}+c_{2} f_{2} \in \Pi$. Let $f$ be a complex function defined and continuous on $S$, and let $p$ be an element of $\Pi$ such that $p(z) \neq f(z)$ throughout $S$. A necessary and sufficient condition for the existence of $a q \in \Pi$ satisfying throughout $S$

$$
|f(z)-q(z)|<|f(z)-p(z)|
$$

is the existence of an $r \in \Pi$, satisfying throughout $S$

$$
|f(z)-p(z)-r(z)|<|f(z)-p(z)+r(z)|
$$

Proof of Lemma 2.

Necessity. Let $r=q-p$. Then throughout $S$

$$
\begin{gathered}
|f(z)-p(z)-r(z)|<|f(z)-p(z)|<|f(z)-p(z)|\{2-|f(z)-q(z)| \times \\
\left.|f(z)-p(z)|^{-1}\right\} \leq|2\{f(z)-p(z)\}-\{f(z)-q(z)\}|=|f(z)-p(z)+r(z)| .
\end{gathered}
$$

Sufficiency. We use the fact that if $a, b$ are arbitrary complex numbers, the inequalities $|a-b|<|a+b|, \operatorname{Re}(b \bar{a})>0$, are equivalent. Since throughout $S$

$$
\operatorname{Re}[r(z) /\{f(z)-p(z)\}]=|f(z)-p(z)|^{-2} \operatorname{Re}[r(z)\{\overline{f(z)-p(z)\}}]>0,
$$

we have there $\alpha|r(z) /\{f(z)-p(z)\}|^{2}<2 \operatorname{Re}[r(z) /\{f(z)-p(z)\}]$ where $\alpha=\min \left[|\{f(z)-p(z)\} / r(z)|{ }^{2} \operatorname{Re}(r(z) /\{f(z)-p(z)\}), z\right.$ on $\left.S\right]$. Let $q=p+\alpha r$. Then throughout $S$,

$$
\begin{gathered}
|f(z)-q(z)|=|f(z)-p(z)|\left|1-\alpha r(z)\{f(z)-p(z)\}^{-1}\right|=|f(z)-p(z)| \times \\
{\left[1+\alpha^{2}|r(z) /(f(z)-p(z))|^{2}-2 \alpha \operatorname{Re}\left\{r(z)(f(z)-p(z))^{-1}\right\}\right]^{1 / 2}<|f(z)-p(z)|}
\end{gathered}
$$


Lemma 3. Let the Hypotheses 1, 2 of Theorem 1 hold, and let $p$ be an element of $I I$ such that $p(z) \neq f(z)$ throughout $S$. For every $z \in S$, let $F(z)$ denote the point $\left(x_{1}(z), y_{1}(z), x_{2}(z), y_{2}(z), \cdots, x_{n}(z), y_{n}(z)\right)$ of the (real) Euclidean $2 n$-space $E_{2 n}$, where $x_{\nu}(z)$ is the real part and $y_{\nu}(z)$ the imaginary part of $\left.p_{\nu}(z) \overline{\{f(z)-p(z)}\right\}$. A necessary and sufficient condition for the existence of a $q \in \Pi$ satisfying (2) throughout $S$, is that the point $\Omega_{2 n}=(0,0, \cdots 0)$ of $E_{2 n}$ does not belong to. the convex hull $H$ of ${ }^{7} F(s)$.

\section{Proof of Lemma 3.}

Necessity. By Lemma 2 there exists an $r \in \Pi$ such that (3), i.e. the inequality

$$
\operatorname{Re}[r(z) \overline{\{f(z)-p(z)}\}]>0
$$

holds throughout $S$. Let $s_{1}, t_{1}, s_{2}, t_{2}, \cdots, s_{n}, t_{n}$ be reals such that throughout $S, r(z)=\sum_{\nu=1}^{n}\left(s_{\nu}-i t_{\nu}\right) p_{\nu}(z)$. Then throughout $S$ we have

$$
\sum_{\nu=1}^{n} s_{\nu} x_{\nu}(z)+t_{\nu} y_{\nu}(z)>0
$$

and thus $F(s)$ is a subset of the half-space

$$
s_{1} x_{1}+t_{1} x_{2}+\cdots+s_{n} x_{2 n-1}+t_{n} x_{2 n}>0 .
$$

Therefore $H$ is also a subset of this half-space, and consequently $\Omega_{2 n} \notin H$.

Sufficiency. Since $H$ is compact and $\Omega_{2 n} \notin H$, we can find a halfspace (5) containing $F(S)$. Thus (4) holds for every $z \in S$. Setting $r=\sum_{\nu=1}^{n}\left(s_{\nu}-i t_{\nu}\right) p_{\nu}$, we have throughout $S$, (3a), and therefore (3). Thus, by Lemma 2, there exists a $q \in \Pi$ satisfying (2) throughout $S$.

Proof of Theorem 1. $f$ cannot belong to $\Pi$, for otherwise, by Lemma 1, the restrictions of $f$ and of $p$ to $S$ would coincide, contradicting Hypothesis 3. By Definition 1, there does not exist a $q \in \Pi$ satisfying (2) throughout $S$. Using notations of the last lemma, it follows that $\Omega_{2 n} \in H$. By a well known theorem of Carathéodory there exist in $F(S)$ distinct points $A_{1}, A_{2}, \cdots, A_{m}(m \leq 2 n+1)$ and there exist positive $A_{1}, A_{2}, \cdots, A_{m}$ such that

$$
\Omega_{2 n}=\sum_{\mu=1}^{m} \Lambda_{\mu} A_{\mu} .
$$

Let

$7 F(s)$ is, as usual, the set of all $F(z), z \in S$. 


$$
A_{\mu}=F\left(z_{\mu}\right), z_{\mu} \in S
$$$$
(\mu=1,2, \cdots, m) .
$$

Then the $z_{\mu}$ are distinct, and from (6) we get by taking components,

$$
\sum_{\mu=1}^{m} A_{\mu} p_{\nu}\left(z_{\mu}\right)\left\{\overline{f\left(z_{\mu}\right)-p\left(z_{\mu}\right)}\right\}=0 \quad(\nu=1,2, \cdots, n) .
$$

"Thus

$$
\sum_{\mu=1}^{m} \lambda_{\mu} p_{\nu}\left(z_{\mu}\right) /\left\{f\left(z_{\mu}\right)-p\left(z_{\mu}\right)\right\}=0 \quad(\nu=1,2, \cdots, n)
$$

where $\lambda_{\mu}=\Lambda_{\mu}\left|f\left(z_{\mu}\right)-p\left(z_{\mu}\right)\right|^{2}>0 \quad(\mu=1,2, \cdots, m)$. Let $s=\left\{z_{1}, z_{2}\right.$, $\left.\cdots, z_{m}\right\}$, and let $\pi$ be the set of all functions defined on $s$ which can be represented throughout $s$ as linear combinations (with complex coefficients) of the $p_{\nu}$. Obviously $p \in \pi$, since $p \in \Pi$. From (6) and (7) it follows that $\Omega_{2 n}$ belongs to the convex hull of $F(s)$ and therefore, by Lemma 3 (taking there $s$ in place of $S$ and $\pi$ in place of $\Pi$ ) there does not exist a $q \in \pi$ satisfying (2) throughout $s$. This concludes the proof.

REMARK 2. Suppose that one of the $p_{\nu}$ in Theorem 1 equals throughout $S$ a constant $c(\neq 0)$. Then from (8) we obtain $\sum_{\mu=1}^{m} \Lambda_{\mu}\left\{f\left(z_{\mu}\right)\right.$ $\left.-p\left(z_{\mu}\right)\right\}=0$. Thus 0 belongs to the convex hull of the image of $s$ (and a fortiori of $S$ ) under $f-p$. [Compare Motzkin and Walsh 1953, $\S 2$, and Fekete 1955, §18].

REMARK 3. Let $s^{\prime}=\left\{z_{1}, z_{2}, \cdots, z_{M}\right\}$ be a finite set in the complex plane and suppose that $f, p_{1}, p_{2}, \cdots, p_{n}$ are complex functions defined on $s^{\prime}$. Let $\pi^{\prime}$ be the set of all complex functions representable throughout $s^{\prime}$ as a linear combination with complex coefficients of $p_{1}, p_{2}, \cdots$, $p_{n}$. Let $p$ be an element of $\pi^{\prime}$ such that $p(z) \neq f(z)$ throughout $s^{\prime}$, and suppose there exist nonnegative reals $\lambda_{1}^{\prime}, \cdots, \lambda_{M}^{\prime}$ (not all zero) such that

$$
\sum_{\mu=1}^{M} \lambda_{\mu}^{\prime} p_{\nu}\left(z_{\mu}\right) /\left\{f\left(z_{\mu}\right)-p\left(z_{\mu}\right)\right\}=0 \quad(\nu=1,2, \cdots, n) .
$$

Then there does not exist a $q \in \pi^{\prime}$ such that (2) holds throughout $s^{\prime}$. Indeed, we have

$$
\sum_{\mu=1}^{M} \Lambda_{\mu}^{\prime} p_{\nu}\left(z_{\mu}\right)\left\{\overline{f\left(z_{\mu}\right)-p\left(z_{\mu}\right)}\right\}=0 \quad(\nu=1,2, \cdots, n)
$$

where $\Lambda_{\mu}^{\prime}$ are nonnegative reals, not all zero. Therefore (using notations of Lemma 3) $\Omega_{2 n}$ belongs to the convex hull of $F\left(s^{\prime}\right)$. By Lemma 3 , there does not exist a $q \in \pi^{\prime}$ satisfying (2) throughout $s^{\prime}$. Consequently, $p$ is a juxtafunction to $f$ on $s^{\prime}$ with respect to $\pi^{\prime}$. 
THEOREM 2. Let the hypotheses of Theorem 1 hold and suppose furthermore that $f-p, p_{1}, p_{2}, \cdots ; p_{n}$ are real valued throughout $S$. Then the inequality $1 \leq m \leq 2 n+1$ in the conclusion of Theorem 1 can be replaced by $1 \leq m \leq n+1$.

Theorem 2 is proved with the aid of the following lemma, in the same way that Theorem 1 was proved with the aid of Lemma 3 .

Lemma 4. Let the hypotheses 1, 2 of Theorem 1 hold, let $p$ be an element of $I I$ such that $f(z) \neq p(z)$ throughout $S$, and suppose that $f-p, p_{1}, p_{2}, \cdots, p_{n}$ are real throughout $S$. For every $z \in S$, let $F_{1}(z)$ denote the point $\left(p_{1}\right)(z)\{f(z)-p(z)\}, p_{2}(z)\{f(z)-p(z)\}, \cdots, p_{n}(z)\{f(z)-$ $p(z)\})$ of the (real) Euclidean $n$-space $E_{n}$. A necessary and sufficient condition for the existence of a $q \in I I$ satisfying (2) throughout $S$, is that the point $\Omega_{n}=(0,0, \cdots, 0)$ of $E_{n}$ does not belong to the convex hull of $F_{1}(S)$.

The proof of the last lemma is analogous to that of Lemma 3.

We shall make frequent use of the concept of unisolvence. We mention therefore the following

DEFINITION 2. Let $S$ be a set in the complex plane, and $\left(p_{\nu}(z)\right)_{\nu=1}^{n}$ a finite sequence of complex functions defined on $S$. The sequence will be called unisolvent on $S$ if and only if for every complex $c_{1}, c_{2}, \cdots, c_{n}$ (not all zero) the set of all $z \in S$ for which $\sum_{v=1}^{n} c_{\nu} p_{\nu}(z)=0$, contains less than $n$ points.

REMARK 4. Thus $\left(p_{\nu}(z)\right)_{\nu=1}^{n}$ is unisolvent on $S$ if and only if this sequence is linearly independent on every $n$-point subset of $S . \quad A$ simple example is the sequence $\left(z^{\nu-1}\right)_{\nu=1}^{n}$, which is unisolvent on every subset of the complex plane. A unisolvent sequence has been termed also (for an important particular case) a "Tchebycheff system". Other terms used in this connection are "Haar system" and "interpolational system".

Theorem 3. Let the hypotheses of Theorem 1 hold and suppose that each of the sequences $\left(p_{\nu}(z)\right)_{\nu=1}^{j}(j=1,2, \cdots, n)$ is unisolvent on S. Then the inequalities

$$
1 \leq m \leq 2 n+1
$$

in Theorem 1, can be replaced by the sharper estimate $n+1 \leq m \leq$ $2 n+1$. Furthermore, if the additional hypothesis of Theorem 2 is 
made too, (9) can be replaced by $m=n+1$.

Proof. Choose distinct points $z_{1}, z_{2}, \cdots, z_{m}$ of $S$ and positive $\lambda_{1}$, $\lambda_{2}, \cdots, \lambda_{m}$ such that (I), (II) and (III) of Theorem 1 hold, where $1 \leq$ $m \leq 2 n+1$ and where, furthermore, $1 \leq m \leq n+1$ in case the additional hypothesis of Theorem 2 holds. We shall prove that $n+1 \leq m$. Indeed: suppose $m \leq n$. Then since $\left(p_{\nu}(z)\right)_{\nu=1}^{m}$ is unisolvent on $S$, the determinant whose $j$ th row is $p_{1}\left(z_{j}\right) p_{2}\left(z_{j}\right) \cdots p_{m}\left(z_{j}\right)$ is different from zero. Therefore there exist constants $c_{1}, \cdots, c_{m}$ such that $f(z)=$ $\sum_{\nu=1}^{n} c_{\nu} p_{\nu}(z)$ throughout $s$. Let $\pi$ have the same meaning as in the proof of Theorem 1 ; then $f \in \pi$. By Theorem 1, (II), $p$ is a juxtafunction to $f$ on $s$ with respect to $\pi$. By Lemma 1 (with $S$ replaced by $s, \Pi$ by $\pi$, and $f$ by the restriction of our $f$ to $s$ ) we have $f(z)=p(z)$ throughout $s$, contradicting hypothesis 3 of Theorem 1 .

2. We apply now Theorems 1,2 and 3 to $n$th infrapolynomials (cf. the Introduction).

THEOREM 4. Let $n$ and $q$ be natural numbers $(q \leq n), n_{1}, n_{2}$, $\cdots, n_{q}$ integers such that $0 \leq n_{1}<n_{2} \cdots<n_{q} \leq n$, and $S$ a closed and bounded set in the complex plane. Let $A(z)(\neq 0$ throughout $S)$ be an $n$th infrapolynomial on $S$ with respect to $\left(n_{1}, \cdots, n_{q}\right)$. Then ${ }^{8}$ there exist distinct points $z_{1}, z_{2}, \cdots, z_{m}$ of $S$,

$$
1 \leq m \leq 2(n-q)+3
$$

and positive $\lambda_{1}, \lambda_{2}, \cdots, \lambda_{m}$ such that $A(z)$ is an $n$th infrapolynomial on $s=\left\{z_{1}, z_{2}, \cdots, z_{m}\right\}$ with respect to $\left(n_{1}, n_{2}, \cdots, n_{q}\right)$ and such that

$$
\sum_{\mu=1}^{m} \lambda_{\mu} z_{\mu}^{l_{\nu}} / A\left(z_{\mu}\right)=0 \quad(\nu=1,2, \cdots, n+1-q)
$$

where $l_{1}, l_{2}, \cdots, l_{n+1-q}\left(l_{1}<l_{2} \cdots<l_{n+1-q}\right)$ are the elements of $\{0,1$, $\cdots, n\}-\left\{n_{1}, n_{2}, \cdots, n_{q}\right\}$. If the polynomials $A(z), z^{l_{1}}, \cdots, z^{l_{n+1-q}}$ are real valued throughout $S$, then (10) can be replaced by $1 \leq m \leq n+$ $2-q$. If each of the sequences $\left(z^{l_{\nu}}\right)_{\nu=1}^{j}(j=1,2, \cdots, n+1-q)$ is unisolvent on $S$, then (10) can be replaced by

$$
n-q+2 \leq m \leq 2(n-q)+3 .
$$

If the polynomials $A(z), z^{l_{1}}, \cdots, z^{l_{n+1-q}}$ are real valued throughout $S$ and each of the sequences $\left(z^{l_{\nu}}\right)_{\nu=1}^{j}(j=1,2, \cdots, n+1-q)$ is unisolvent on $S$, then (10) can be replaced by $m=n-q+2$.

REMARK 5. If $\left(n_{1}, n_{2}, \cdots, n_{q}\right)$ of Theorem 4 is a simple $n$-sequ-

8 As is easily seen, $S$ cannot be empty. [Cf. Shisha and Walsh, 1961, footnote 7 on p. 117]. 
ence (cf. the Introduction) and if, in case $n_{1}=0,0 \notin S$, then as is easily seen, the sequences $\left(z^{l_{\nu}}\right)_{\nu=1}^{j}(j=1,2, \cdots, n+1-q)$ are unisolvent on $S$.

Proof of Theorem 4. Let $I$ be the set of all complex functions defined on $S$ which are expressible throughout $S$ as linear combinations of $z^{l_{1}}, z^{l_{2}}, \cdots, z^{l_{n+1-q}}$ with complex coefficients, and let $f(z) \equiv \sum_{\nu=1}^{q} a_{n_{\nu}} z^{n_{\nu}}$, $p(z) \equiv-\sum_{\nu=1}^{n+1-q} a_{l_{\nu}} z^{l_{\nu}}$. It is easily seen that $p(z)$ is a juxtafunction to $f$ on $S$ with respect to $\Pi$. Therefore, by Theorem 1 there exist distinct points $z_{1}, \cdots, z_{m}(m \leq 2(n+1-q)+1=2(n-q)+3)$ of $S$ and positive $\lambda_{1}, \lambda_{2}, \cdots, \lambda_{m}$ such that (11) holds, and such that no complex $b_{1}, b_{2}, \cdots, b_{n+1-q}$ exist satisfying

$$
\left|\sum_{\nu=1}^{q} a_{n_{\nu}} z^{n_{\nu}}-\sum_{\nu=1}^{n+1-q} b_{\nu} z^{l_{\nu}}\right|<|A(z)|
$$

throughout $s=\left\{z_{1}, z_{2}, \cdots, z_{m}\right\}$. Thus $A(z)$ is an $n$th infrapolymial on $s$ with respect to $\left(n_{1}, n_{2}, \cdots, n_{q}\right)$. The rest of Theorem 4 follows from Theorems 2 and 3.

REMARK 6 . Let $n, n_{1}, n_{2}, \cdots, n_{q}$ be integers $\left(q \leq n, 0 \leq n_{1}<n_{2}\right.$ $\left.\cdots<n_{q} \leq n\right), \quad A(z) \equiv \sum_{1 \gamma=0}^{n} a_{\nu} z^{\nu}$ a polynomial, $z_{1}, z_{2}, \cdots, z_{M}$ points of the complex plane, and $\lambda_{1}^{\prime}, \lambda_{2}^{\prime}, \cdots, \lambda_{M}^{\prime}\left(\sum_{\mu=1}^{M} \lambda_{\mu}^{\prime}>0\right)$ nonnegative reals such that $A\left(z_{\mu}\right) \neq 0(\mu=1,2, \cdots, M)$, and such that $\sum_{\mu=1}^{M} \lambda_{\mu}^{\prime} z_{\mu}^{l \nu} / A\left(z_{\mu}\right)$ $=0 \quad(\nu=1,2, \cdots, n+1-q)$, where the $l_{\nu}$ have the same meaning as in Theorem 4. Then $A(z)$ is an $n$th infrapolynomial on $s^{\prime}=\left\{z_{1}, z_{2}\right.$, $\left.\cdots, z_{M L}\right\}$ with respect to $\left(n_{1}, n_{2}, \cdots, n_{q}\right)$. Indeed: let $f$ and $p$ be as in the last proof, and let $\pi^{\prime}$ be the set of all complex functions representable throughout $s^{\prime}$ as a linear combination (with complex coefficients) of $z^{l_{1}}, z^{l_{2}}, \cdots, z^{l_{n+1-q}}$. The asserted conclusion follows from Remark 3 .

We give now the following structure theorem which is the main result of this paper.

THEOREM 5. Let $n$ and $q(1 \leq q \leq n)$ be integers, and $\sigma$ a simple $n$-sequence of $q$ elements. Let $S$ be a closed and bounded set in the complex plane, and in case $0 \in \sigma$, assume that $0 \notin S$. Let $A(z)(\neq \equiv)$ be an nth infrapolynomial on $S$ with respect to $\sigma$, and let $B(z)(\neq 0$ throughout $S$ ) be a divisor of $A(z)$. Assume also that the degree $r$ of $B(z)$ is $\geq q$. Then $B(z)$ is a divisor of some

$$
Q(z) \equiv P(z) g(z)+z^{K} \sum_{\mu=1}^{M-q+2} \lambda_{\mu} g(z) /\left(z-z_{\mu}\right) .
$$

$9 \quad$ By degree of a polynomial $(\not \equiv 0)$ we mean its exact degree. The polynomial 0 is assigned the degree-1. 
Here $M$ is an integer satisfying $r \leq M \leq 2 r-q+1$, the $z_{\nu}$ are distinct points of $S, g(z) \equiv \prod_{\mu=1}^{\mathcal{M}-q+2}\left(z-z_{\mu}\right)$, the $\lambda_{\mu}$ are positive reals with $\sum_{\mu=1}^{M-q+2} \lambda_{\mu}=1, P(z)$ is a polynomial of degree $\leq q-1$ such that $P(z) g(z)+z^{K+M-q+1}$ is of degree $\leq M$, and $K$ is $\min [\nu, \nu \notin \sigma, \nu=0,1,2, \cdots]$.

REMARK 7. As will be seen from the proof of Theorem 5, if $S$ and the coefficients of $B(z)$ are real, the inequality $r \leq M \leq 2 r-q+1$ of the theorem can be replaced by the equality $M=r$.

In the proof of Theorem 5 use will be made of the following

LEMMA 5. Let $n, q, \sigma$ and $K$ be as in the last theorem, let $S$ be $a$ set in the complex plane, and let $A(z)(\not \equiv 0)$ be an $n$th infrapolynomial on $S$ with respect to $\sigma$. Let $B(z)$ be a polynomial of degree $r(\geq q)$ dividing $A(z)$. Then $B(z)$ is an $r$ th infrapolynomial on $S$ with respect to $\sigma_{0}$, where $\sigma_{0}$ is that simple r-sequence of $q$ elements for which $K=\min \left[\nu, \nu \notin \sigma_{0}, \nu=0,1,2, \cdots\right]$.

The proof of Lemma 5 is straightforward and may be omitted.

Proof of Theorem 5. By Lemma 5, $B(z)$ in an $r$ th infrapolynomial on $S$ with respect to the sequence $\sigma_{0}$ defined there. We choose (cf. Theorem 4 and Remark 5) distinct points $z_{1}, z_{2}, \cdots, z_{m}$ of $S$ and positive $\lambda_{1}, \lambda_{2}, \cdots, \lambda_{m}$ such that $\sum_{\mu=1}^{m} \lambda_{\mu}=1$ and

$$
\sum_{\mu=1}^{m} \lambda_{\mu} z_{\mu}^{\rho} / B\left(z_{\mu}\right)=0
$$

for every integer $\rho$ satisfying $0 \leq \rho \leq r, \rho \notin \sigma_{0}$. Here $m$ is an integer satisfying $r-q+2 \leq 2(r-q)+3$, and in case $S$ and the coefficients of $B(z)$ are real we may take $m=r-q+2$. Set

$$
g(z) \equiv \prod_{\mu=1}^{m}\left(z-z_{\mu}\right), \quad N(z) \equiv \sum_{\mu=1}^{m} \lambda_{\mu} z_{\mu}^{r-q+K+1} g(z) /\left\{B\left(z_{\mu}\right)\left(z-z_{\mu}\right)\right\} .
$$

If $\mu$ and $\nu$ are integers, $1 \leq \mu \leq m, 0 \leq \nu \leq r-q+K$, then

$$
\left[\lambda_{\mu} z_{\mu}^{r-q+K+1} g(z) /\left\{B\left(z_{\mu}\right)\left(z-z_{\mu}\right)\right\}\right]_{z=0}^{(\nu)}=-\sum_{j=0}^{\nu} \lambda_{\mu} z_{\mu}^{r-q+K-j}\left(\begin{array}{l}
\nu \\
j
\end{array}\right) j ! g^{(\nu-j)}(0) / B\left(z_{\mu}\right)
$$

(the equality is obvious if $z_{\mu}=0$, and otherwise it is obtained by Leibnitz's rule for differentiating a product). Therefore, from (15) we get

$$
\begin{gathered}
N^{(\nu)}(0)=-\sum_{j=0}^{\nu}\left(\begin{array}{l}
\nu \\
j
\end{array}\right) j ! g^{(\nu-j)}(0) \sum_{\mu=1}^{m} \lambda_{\mu} z_{\mu}^{r-q+K-j} / B\left(z_{\mu}\right) \\
(\nu=0,1, \cdots, r-q+K) .
\end{gathered}
$$


Since $\{0,1, \cdots, r\}-\left\{\sigma_{0}\right\}=\{r-q+K-j\}_{j=0}^{r-q}$, therefore (16) and (14) yield $N^{(\nu)}(0)=0, \nu=0,1, \cdots, r-q$. Hence we can write $N(z) \equiv z^{r-q+1} M_{1}(z)$, where $M_{1}(z)$ is a polynomial (of degree $\leq m-2$ ). Let

$$
M_{2}(z) \equiv \sum_{\mu=1}^{m} \lambda_{\mu} z_{\mu}^{K} g(z) /\left\{B\left(z_{\mu}\right)\left(z-z_{\mu}\right)\right\} .
$$

By (14),

$$
\sum_{\mu=1}^{m} \lambda_{\mu} z_{\mu}^{K} / B\left(z_{\mu}\right)=0
$$

and therefore the degree of $M_{2}(z)$ is $\leq m-2$. For every $z_{j}$ different from zero we have by (15), $M_{1}\left(z_{j}\right)=z_{j}^{-r+q-1} N\left(z_{j}\right)=\lambda_{j} z_{j}^{K} g^{\prime}\left(z_{j}\right) / B\left(z_{j}\right)=$ $M_{2}\left(z_{j}\right)$. Since there are at least $m-1$ such $z_{j}$, we have $M_{1}(z) \equiv$ $M_{2}(z)$. Consider now the polynomial

$$
R(z) \equiv B(z) M_{2}(z)-\sum_{\mu=1}^{m} \lambda_{\mu} z_{\mu}^{K} g(z) /\left(z-z_{\mu}\right) .
$$

For $j=1,2, \cdots, m$ we have $R\left(z_{j}\right)=B\left(z_{j}\right) M_{2}\left(z_{j}\right)-\lambda_{j} z_{j}^{K} g^{\prime}\left(z_{j}\right)=0$. Therefore we can write $R(z) \equiv g(z) U(z)$, where $U(z)$ is some polynomial. Also, the relation $N(z) \equiv z^{r-q+1} M_{2}(z)$ and the definition of $R(z)$ imply that the degree of the latter is $\leq m+q-2$. Therefore the degree: of $U(z)$ is at most $q-2$. If $K \geq 1$, then the relation

$$
B(z) M_{2}(z) \equiv g(z) U(z)+\sum_{\mu=1}^{m} \lambda_{\mu} z_{\mu}^{K} g(z) /\left(z-z_{\mu}\right)
$$

yields, upon putting $z_{\mu}^{K}=\left[z+\left(z_{\mu}-z\right)\right]^{K}$ and developing the last right member,

$$
B(z) M_{2}(z) \equiv g(z)\left[U(z)+A_{K-1}(z)\right]+z^{K} \sum_{\mu=1}^{m} \lambda_{\mu} g(z) /\left(z-z_{\mu}\right),
$$

where $A_{K-1}(z)$ is a polynomial of degree $K-1$. The last relation (with $\left.A_{K-1}(z) \equiv 0\right)$ holds also when $K=0$. We set now $P(z) \equiv U(z)+A_{K-1}(z)$, and get that $B(z)$ is a divisor of

$$
Q(z) \equiv P(z) g(z)+z^{K} \sum_{\mu=1}^{m} \lambda_{\mu} g(z) /\left(z-z_{\mu}\right) .
$$

The degree of $Q(z)$, i. e. of $B(z) M_{2}(z)$, is $\leq m+q-2$. Thus the degree of $P(z)$ is $\leq q-1$, and that of $P(z) g(z)+z^{K+m-1}$ is $\leq m+q-2$. We set now $M=m+q-2$, and observe that the conclusions of the theorem are all satisfied.

REMARK 8. The polynomial $Q(z)$ of (13) is an $M$ th infrapolynomial on $\left\{z_{1}, z_{2}, \cdots, z_{M-q+2}\right\}$ with respect to $\sigma_{1}$, where $\sigma_{1}$ is that simple 
$M$-sequence of $q$ elements for which $\min \left[\nu, \nu \notin \sigma_{1}, \nu=0,1,2, \cdots\right]=K$. This follows from Theorem 1 of Shisha and Walsh [1961].

THEOREM 6. Let $S$ be a closed and bounded set in the complex plane, $A(z) \equiv \sum_{y=0}^{n} a_{\gamma} z^{\nu}\left(n \geq 1, a_{n} \neq 0\right)$ an $n$th infrapolynomial on $S$ with respect to $(n-1)$, and suppose that $A(z) \neq 0$ throughout $S$. Then:

(a) Every zero $\zeta$ of $A(z)$ is of the form

$$
c(\zeta)-\lambda(\zeta)\left[a_{n-1} / a_{n}\right]
$$

where $c(\zeta)$ belongs to the convex hull of $S$ and where $0 \leq \lambda(\zeta) \leq 10^{10}$

(b) Suppose that $S$ lies in a closed disc $C:|z-a| \leq r(\geq 0)$. Then all zeros of $A(z)$ belong to $C \cup C_{1}$, where $C_{1}$ is the closed disc $\left|z-\left[a-\left(a_{n-1} / a_{n}\right)\right]\right| \leq r$. If $C$ and $C_{1}$ are disjoint then $A(z)$ has at least $n-1$ zeros belonging to $C$. [Multlplicities are always being counted].

Proof. We choose distinct points $z_{1}, z_{2}, \cdots, z_{m}$ of $S$ and positive $\lambda_{1}, \lambda_{2}, \cdots, \lambda_{m}(m \leq 2 n+1)$ such that $\sum_{\mu=1}^{m} \lambda_{\mu}=1$ and $\sum_{\mu=1}^{m} \lambda_{\mu} z_{\mu}^{\rho} / A\left(z_{\mu}\right)=0$ for all integers $\rho$ with $0 \leq \rho \leq n, \rho \neq n-1$. Then $1=\sum_{\mu=1}^{m} \lambda_{\mu} A\left(z_{\mu}\right) /$ $A\left(z_{\mu}\right)=\sum_{\mu=1}^{m} \lambda_{\mu} a_{n-1} z_{\mu}^{n-1} / A\left(z_{\mu}\right)$, and so

$$
\sum_{\mu=1}^{m} \lambda_{\mu} z_{\mu}^{n-1} / A\left(z_{\mu}\right)=1 / a_{n-1} .
$$

We $\quad$ set $\quad g(z) \equiv \prod_{\mu=1}^{m}\left(z-z_{\mu}\right), \quad N(z) \equiv \sum_{\mu=1}^{m} \lambda_{\mu} z_{\mu}^{n-1} g(z) /\left\{A\left(z_{\mu}\right)\left(z-z_{\mu}\right)\right\} \equiv$ $a_{n-1}^{-1} z^{m-1}+\cdots$. We follow the proof of Theorem 5 from the sentence following (15). Again we have $N^{(v)}(0)=0$ for every $\nu$ satisfying $0 \leq$ $\nu \leq n-2$. Thus we may set $N(z) \equiv z^{n-1} M_{1}(z)$, where $M_{1}(z) \equiv a_{n-1}^{-1} z^{m-n}+\cdots$ is some polynomial. Let $M_{2}(z) \equiv \sum_{\mu=1}^{m} \lambda_{\mu} g(z) /\left\{A\left(z_{\mu}\right)\left(z-z_{\mu}\right)\right\}$. If $n=1$, then $M_{2}(z) \equiv N(z) \equiv M_{1}(z)$. If $n>1$ then for each $z_{j}$ different from zero, $M_{1}\left(z_{j}\right)=\lambda_{\mu} g^{\prime}\left(z_{j}\right) / A\left(z_{j}\right)=M_{2}\left(z_{j}\right)$, and since there are at least $m-1$ such $z_{j}$ and $M_{1}(z)$ and $M_{2}(z)$ are of degrees $\leq m-2$, we have again $M_{2}(z) \equiv M_{1}(z)$. Consider now the polynomial $R(z) \equiv A(z) M_{2}(z)-$ $\sum_{\mu=1}^{m} \lambda_{\mu} g(z) /\left(z-z_{\mu}\right) \equiv\left(a_{n} / a_{n-1}\right) z^{m}+\cdots$ For $j=1,2, \cdots, m, R\left(z_{j}\right)=0$, and therefore $R(z) \equiv\left(a_{n} / a_{n-1}\right) g(z)$. Thus, $A(z)$ is a divisor of $Q(z) \equiv$ $\left(a_{n} / a_{n-1}\right) g(z)+\sum_{\mu=1}^{m} \lambda_{\mu} g(z) /\left(z-z_{\mu}\right)$. Let $\zeta$ be a zero of $A(z)$. Then $g(\zeta) \neq 0$, and thus $a_{n} / a_{n-1}+\sum_{\mu=1}^{m} \lambda_{\mu} /\left(\zeta-z_{\mu}\right)=0$. Since $\sum_{\mu=1}^{m} \lambda_{\mu} /\left(\zeta-z_{\mu}\right)$ can be written [Shisha and Walsh 1961, Lemma on p. 127] as $\lambda(\zeta) /$ $(\zeta-c(\zeta))$ where $c(\zeta)$ and $\lambda(\zeta)$ are as required in (a) of our theorem, $\zeta$ is of the form (17). Suppose now that $S$ lies in a closed disc $C:|z-\alpha|$ $\leq r(\geq 0)$. Then by a theorem due to J. L. Walsh [cf. 1922, Theorem VI; see also Shisha and Walsh 1961, p. 147] all zeros of $Q(z)$ lie in

10 Thus $\zeta$ belongs to the set swept by the convex hull of $S$ while being displaced, the displacement being given by the vector $-a_{n-1} / a_{n}$. 
$C \cup C_{1}$, and if $C$ and $C_{1}$ are disjoint, the number of zeros of $Q(z)$ in them is, respectively, $m-1$ and 1 . From this follow the conclusions of part (b) of our theorem.

\section{BIBLIOGRAPHY}

1. M. Fekete 1951. On the structure of extremal polynomials, Proc. Natl. Acad. Sci. U.S. 37, pp. 95-103. 1955. On the structure of polynomials of least deviation, Bull. Research Council Israel 5A, pp. 11-19.

2. T. S. Motzkin and J. L. Walsh 1953. On the derivative of a polynomial and Chebychev approximation, Proc. Amer. Math. Soc. 4, pp. 76-87.

3. O. Shisha 1958. Properties of extremal systems of points (mimeographed), Ph. D. thesis. 1958a. Nearest functions (abstract), Notices Amer. Math. Soc. 5, No. 7, p. 853. 1959. On generalized infrapolynomials of a certain type (abstract), Ibid. 6, No. 3, p. 283. 1961. A structure theorem for infrapolynomials with prescribed coefficients (abstract), Ibid. 8, No. 1, p. 66.

4. O. Shisha and J. L. Walsh 1961. The zeros of infrapolynomials with some prescribed coefficients, J. d'analyse Math. 9, pp. 111-160.

5. J. L. Walsh 1922. On the location of the roots of certain types of polynomials, Trans. Amer. Math. Soc. 24, pp. 163-180. 1958. On infrapolynomials with prescribed constant term, J. Math. Pures Appl. 37, pp. 295-316.

6. J. L. Walsh and T. S. Motzkin 1957. Polynomials of best approximation on a real finite point set, Proc. Natl. Acad. Sci. U. S. 43, pp. 845-846.

7. J. L. Walsh and M. Zedek 1956. On generalized Tchebycheff polynomials, Ibid. 42, pp. 99-104.

Aerospace Research Laboratories, Wright-Patterson Air Force Base

HARVARD UNIVERSITY AND

National BUREaU of Standards 



\section{PACIFIC JOURNAL OF MATHEMATICS}

\section{EDITORS}

\author{
Robert Osserman \\ Stanford University \\ Stanford, California
M. G. Arsove
University of Washington
Seattle 5 , Washington

\author{
J. DugundJI \\ University of Southern California \\ Los Angeles 7, California
}

Lowell J. Paige

University of California

Los Angeles 24, California

\section{ASSOCIATE EDITORS}
E. F. BECKENBACH
B. H. NeumanN
F. WOLF
K. YOSIDA

\section{SUPPORTING INSTITUTIONS}

\author{
UNIVERSITY OF BRITISH COLUMBIA \\ CALIFORNIA INSTITUTE OF TECHNOLOGY \\ UNIVERSITY OF CALIFORNIA \\ MONTANA STATE UNIVERSITY \\ UNIVERSITY OF NEVADA \\ NEW MEXICO STATE UNIVERSITY \\ OREGON STATE UNIVERSITY \\ UNIVERSITY OF OREGON \\ OSAKA UNIVERSITY \\ UNIVERSITY OF SOUTHERN CALIFORNIA
}

\author{
STANFORD UNIVERSITY \\ UNIVERSITY OF TOKYO \\ UNIVERSITY OF UTAH \\ WASHINGTON STATE UNIVERSITY \\ UNIVERSITY OF WASHINGTON \\ * * * * \\ AMERICAN MATHEMATICAL SOCIETY \\ CALIFORNIA RESEARCH CORPORATION \\ SPACE TECHNOLOGY LABORATORIES \\ NAVAL ORDNANCE TEST STATION
}

Mathematical papers intended for publication in the Pacific Journal of Mathematics should by typewritten (double spaced), and on submission, must be accompanied by a separate author's résumé. Manuscripts may be sent to any one of the four editors. All other communications to the editors should be addressed to the managing editor, L. J. Paige at the University of California, Los Angeles 24, California.

50 reprints per author of each article are furnished free of charge; additional copies may be obtained at cost in multiples of 50 .

The Pacific Journal of Mathematics is published quarterly, in March, June, September, and December. Effective with Volume 13 the price per volume (4 numbers) is $\$ 18.00$; single issues, $\$ 5.00$. Special price for current issues to individual faculty members of supporting institutions and to individual members of the American Mathematical Society: $\$ 8.00$ per volume; single issues $\$ 2.50$. Back numbers are available.

Subscriptions, orders for back numbers, and changes of address should be sent to Pacific Journal of Mathematics, 103 Highland Boulevard, Berkeley 8, California.

Printed at Kokusai Bunken Insatsusha (International Academic Printing Co., Ltd.), No. 6, 2-chome, Fujimi-cho, Chiyoda-ku, Tokyo, Japan.

PUBLISHED BY PACIFIC JOURNAL OF MATHEMATICS, A NON-PROFIT CORPORATION

The Supporting Institutions listed above contribute to the cost of publication of this Journal, but they are not owners or publishers and have no responsibility for its content or policies. 


\section{Pacific Journal of Mathematics}

\section{Vol. 14, No. $3 \quad$ July, 1964}

Erik Balslev and Theodore William Gamelin, The essential spectrum of a class of ordinary differential operators . . . . . . . . . . . . . . . . . . . .

James Henry Bramble and Lawrence Edward Payne, Bounds for derivatives in

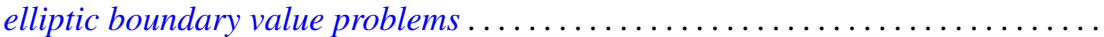

Hugh D. Brunk, Integral inequalities for functions with nondecreasing

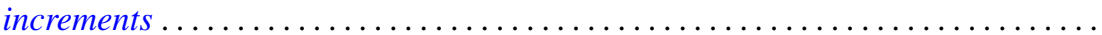

William Edward Christilles, A result concerning integral binary quadratic

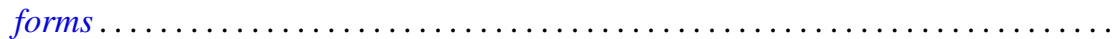

Peter Crawley and Bjarni Jónsson, Refinements for infinite direct decompositions of

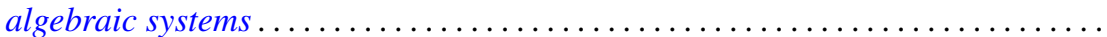

Don Deckard and Carl Mark Pearcy, On continuous matrix-valued functions on a Stonian space.

Raymond Frank Dickman, Leonard Rubin and P. M. Swingle, Another

characterization of the $n$-sphere and related results $\ldots \ldots \ldots \ldots \ldots \ldots$

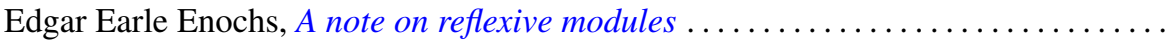

Vladimir Filippenko, On the reflection of harmonic functions and of solutions of the

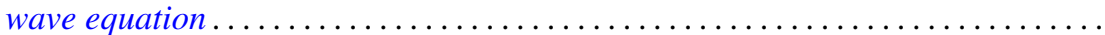

Derek Joseph Haggard Fuller, Mappings of bounded characteristic into arbitrary

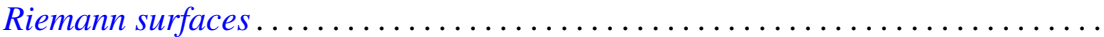
895

Curtis M. Fulton, Clifford vectors . . . . . . . . . . . . . . . . . . . . . . . . . . . . . . 917

Irving Leonard Glicksberg, Maximal algebras and a theorem of Radó . .

919

Kyong Taik Hahn, Minimum problems of Plateau type in the Bergman metric

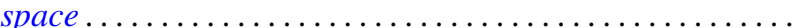

A. Hayes, A representation theory for a class of partially ordered rings...

J. M. C. Joshi, On a generalized Stieltjes trasform

J. M. C. Joshi, Inversion and representation theorems for a generalized Laplace transform ...

Eugene Kay McLachlan, Extremal elements of the convex cone $B_{n}$ of functions ...

Robert Alan Melter, Contributions to Boolean geometry of p-rings ...

James Ronald Retherford, Basic sequences and the Paley-Wiener criterion . . . . . . . 1019

Dallas W. Sasser, Quasi-positive operators. .

Oved Shisha, On the structure of infrapolynomials with prescribed coefficients ..

Oved Shisha and Gerald Thomas Cargo, On comparable means

Maurice Sion, A characterization of weak ${ }^{*}$ convergence ........

Morton Lincoln Slater and Robert James Thompson, A permanent inequality for

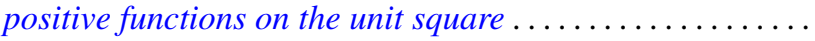

David A. Smith, On fixed points of automorphisms of classical Lie algebras ...

Sherman K. Stein, Homogeneous quasigroups ................

J. L. Walsh and Oved Shisha, On the location of the zeros of some infrapolynomials with prescribed coefficients .

Ronson Joseph Warne, Homomorphisms of $d$-simple inverse semigroups with identity . 